\title{
THE PULSATION OF $\chi$ CYGNI IMAGED BY OPTICAL INTERFEROMETRY: A NOVEL TECHNIQUE TO DERIVE DISTANCE AND MASS OF MIRA STARS
}

\author{
S. Lacour ${ }^{1}$, E. Thiébaut ${ }^{2}$, G. Perrin ${ }^{1}$, S. Meimon $^{3}$, X. Haubois $^{1}$, E. Pedrettir ${ }^{4}$, S. T. Ridghay ${ }^{5}$, \\ J. D. Monnier ${ }^{6}$, J. P. Berger ${ }^{7}$, P. A. Schuller ${ }^{8}$, H. Woodruff ${ }^{9}$, A. Poncelet ${ }^{1}$, H. Le Coroller ${ }^{10}$, R. Millan-Gabet ${ }^{11}$, \\ M. LACASSE ${ }^{12}$, AND W. Traub ${ }^{13}$ \\ 1 Observatoire de Paris, LESIA, CNRS/UMR 8109, 92190 Meudon, France \\ ${ }^{2}$ Centre de Recherche Astrophysique de Lyon, CNRS/UMR 5574, 69561 Saint Genis Laval, France \\ ${ }^{3}$ Office National d'Études et de Recherches Aéronautiques, DOTA, 92322 Chatillon, France \\ ${ }^{4}$ School of Physics and Astronomy, University of St. Andrews, North Haugh, St Andrews KY16 9SS, UK \\ ${ }^{5}$ National Optical Astronomy Observatory, P.O. Box 26732, Tucson, AZ 85726-6732, USA \\ ${ }^{6}$ University of Michigan, Astronomy Department, 914 Dennison Bldg., 500 Church Street, Ann Arbor, MI 40109, USA \\ ${ }^{7}$ LAOG-UMR 5571, CNRS and Université Joseph Fourier, BP 53, 38041 Grenoble, France \\ ${ }^{8}$ Institut d'Astrophysique Spatiale, CNRS/UMR 8617, Université Paris-Sud, 91405 Orsay, France \\ ${ }^{9}$ Sydney Institute for Astronomy (SIfA), School of Physics, University of Sydney, NSW 2006, Australia \\ ${ }^{10}$ Observatoire de Haute-Provence, OHP/CNRS, F-04870 St. Michel l'Observatoire, France \\ ${ }^{11}$ Michelson Science Center, California Institute of Technology, MS 100-22, Pasadena, CA 91125, USA \\ ${ }^{12}$ Harvard-Smithsonian Center for Astrophysics, 60 Garden Street, Cambridge, MA 02138, USA \\ ${ }^{13}$ Jet Propulsion Laboratory, California Institute of Technology, M/S 301-451, 4800 Oak Grove Drive, Pasadena, CA 91109, USA \\ Received 2009 June 22; accepted 2009 October 22; published 2009 November 24
}

\begin{abstract}
We present infrared interferometric imaging of the S-type Mira star $\chi$ Cygni. The object was observed at four different epochs in 2005-2006 with the Infrared-Optical Telescope Array optical interferometer ( $H$ band). Images show up to $40 \%$ variation in the stellar diameter, as well as significant changes in the limb darkening and stellar inhomogeneities. Model fitting gave precise time-dependent values of the stellar diameter, and reveals presence and displacement of a warm molecular layer. The star radius, corrected for limb darkening, has a mean value of 12.1 mas and shows a 5.1 mas amplitude pulsation. Minimum diameter was observed at phase $0.94 \pm 0.01$. Maximum temperature was observed several days later at phase $1.02 \pm 0.02$. We also show that combining the angular acceleration of the molecular layer with $\mathrm{CO}(\Delta v=3)$ radial velocity measurements yields a $5.9 \pm 1.5$ mas parallax. The constant acceleration of the $\mathrm{CO}$ molecules—during $80 \%$ of the pulsation cycle-lead us to argument for a free-falling layer. The acceleration is compatible with a gravitational field produced by a $2.1_{-0.7}^{+1.5}$ solar mass star. This last value is in agreement with fundamental mode pulsator models. We foresee increased development of techniques consisting in combining radial velocity with interferometric angular measurements, ultimately allowing total mapping of the speed, density, and position of the diverse species in pulsation-driven atmospheres.
\end{abstract}

Key words: infrared: stars - stars: AGB and post-AGB - stars: fundamental parameters - stars: individual (chi Cyg) - techniques: interferometric

Online-only material: color figures

\section{INTRODUCTION}

Mira variables are low- to intermediate-mass asymptotic giant branch (AGB) stars that pulsate with a period of about one year. They have a cool $\left(T_{\text {eff }} \leqslant 3000 \mathrm{~K}\right)$ and extended $\left(R>100 R_{\odot}\right)$ photosphere. As such, they are bright $\left(M_{k} \leqslant-7\right)$ infrared beacons, individually observable far into galaxies of the Local Group (Zijlstra 1999). They have the potential to probe places where the distance (e.g., NGC 5128 in Rejkuba 2004) or reddening (e.g., the Galactic Center in Matsunaga et al. 2009) does not allow observation of the fainter/bluer-and rarerCepheids.

However, the challenge to overcome is that Mira stars are both intrinsically complicated and ill-understood. Two important relations are of special interest: the period/luminosity $(\mathrm{P} / \mathrm{L})$ and the period/mass/radius $(\mathrm{P} / \mathrm{M} / \mathrm{R})$. The first relation has been derived from population studies (sequence " $\mathrm{C}$ " in the LMC from Wood 2000 and also in the globular cluster 47 Tuc from Lebzelter \& Wood 2005). The present best parameterization of the $\mathrm{P}-\mathrm{L}$ relation within our galaxy is (Whitelock et al. 2008):

$$
M_{k}=-(3.51 \pm 0.20)(\log (P)-2.38)-(7.25 \pm 0.07),
$$

where $P$ is the period in days. The zero point of this relation is the most uncertain parameter, with its dependence on the metallicity hardly known. The main difficulty is that parallax values are inaccurate and error-prone due to the large size and inhomogeneous surface brightness of the objects.

The second relation, the $\mathrm{P} / \mathrm{M} / \mathrm{R}$ relation, has more relevance to the fundamental physics of the star. It is extremely dependent on the pulsation mode, but also, less crucially, on the surface density and metallicity. The $\mathrm{P} / \mathrm{M} / \mathrm{R}$ relation has been formally derived from numerical modeling of the fundamental pulsation mode of these stars (Wood 1990):

$$
\log (P)=-2.07+1.94 \log \left(R / R_{\odot}\right)-0.9 \log \left(M / M_{\odot}\right),
$$

Twenty years later, this model-derived relationship has still rarely been confronted with observation. This paper is a first step forward to establish the $\mathrm{P} / \mathrm{L}$ and $\mathrm{P} / \mathrm{M} / \mathrm{R}$ relations on a new firm observational footing.

Of the three crucial parameters (distance, mass, and radius), the angular diameter is far from being the easiest value to obtain. Because the surface gravity is several orders of magnitude lower than the Sun, the pulsation of Mira variable leads to an extended 
atmosphere. In the cool upper layers, significant amount of the products of the helium fusion react to form di- and polyatomic molecules including $\mathrm{TiO}, \mathrm{SiO}, \mathrm{CO}$, and $\mathrm{H}_{2} \mathrm{O}$. The forest of molecular lines and scattering from the dust lead to exotic intensity distributions not at all like a simple stellar disk. In the past, this substantially affected many stellar angular diameter measurements (van Belle et al. 1996; Perrin et al. 1999), paving the way to contentious discussion on the mode of pulsation (Barthes 1998; Ya'Ari \& Tuchman 1999).

However, nowadays, interferometers are able to provide maps of the brightness distribution as a function of wavelength (Le Bouquin et al. 2009; Pluzhnik et al. 2009). Images of the Mira star T Lep revealed a shell-like atmosphere, with a bright chromatic zone distinctly detached from the photosphere. This could be the first image of what Tsuji (2000) called the MOLsphere (Ohnaka 2004), a zone of increased density in which formation of warm molecular species would be favored. Accounting for this layer is the key to obtain a correct value for the diameter (Perrin et al. 1999, 2004; Mennesson et al. 2002). We will also show that we can apply to this layer a modified Baade-Wesselink method to derive the distance and mass of the star.

The test star of this paper is $\chi$ Cyg, a S-type Mira star. It has a pulsation period of 408 days, a photometric magnitude ranging from 5.3 to 13.3, and intense emission lines at postmaximum (Merrill 1947). This suggests a large pulsation amplitude. Images were obtained with the Infrared-Optical Telescope Array (IOTA) interferometer at four different stellar phases, chronologically $\phi=0.93,0.26,0.69$, and 0.79 . In the next section, we describe the observations and give a short overview of the data set. In Section 3, we use an image reconstruction algorithm to map the brightness distribution of the star. Precise geometrical parameters of the star, including existence of the molecular layer, are determined by model fitting in Section 4. From these values, temperatures and opacities are deduced in Section 5. Finally, in Section 6, we combine angular acceleration with radial velocities measurements to derive estimations of the distance and mass of the star.

\section{OBSERVATION AND DATA REDUCTION}

The interferometric data presented herein were obtained using the IOTA interferometer (Traub et al. 2003), a long baseline interferometer which operated at near-infrared wavelengths. It consisted of three $0.45 \mathrm{~m}$ telescopes movable among 17 stations along two orthogonal linear arms. IOTA synthesized a total aperture size of $35 \times 15 \mathrm{~m}$, corresponding to an angular resolution of $\approx 10 \times 23$ milliarcsec at $1.65 \mu \mathrm{m}$. Squared visibility and closure phase $(\mathrm{CP})$ measurements were obtained using the integrated optics combiner IONIC (Berger et al. 2003). IOTA ceased operation in 2006 July.

The declination of $\chi$ Cyg made possible observations at low airmass with the IOTA interferometer during six months per year. With a period of 408 days, it allowed observations around both the minimum and the maximum brightnesses. It was observed at four different stellar phases: 2005 May $(\phi=0.93)$; 2005 October $(\phi=0.26) ; 2006$ April $(\phi=0.69)$; and 2006 May $(\phi=0.79)$. Observation information can be found in Table 1, including dates and phase of observation, interferometer configurations, and projected baseline lengths. Figure 1 shows the $u$ - $v$ coverage achieved during these observation runs. The geometry of the IOTA interferometer and the position of the star on the sky constrained the extent of frequency coverage.

The first three periods of observations were undertaken with a narrow $\mathrm{H}$-band filter $(1.59 \mu \mathrm{m} \leqslant \lambda \leqslant 1.69 \mu \mathrm{m})$. A dispersive
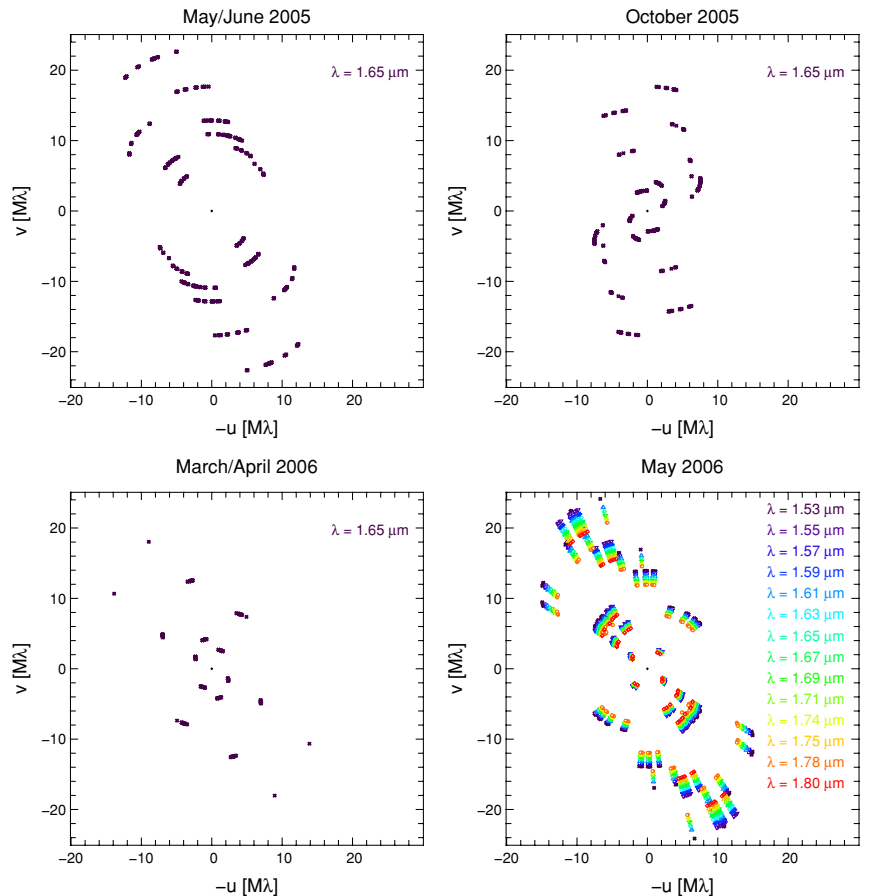

Figure 1. $u-v$ coverage obtained on $\chi \mathrm{Cyg}$ at the four epochs of observation Coordinates are in factors of the wavelength, i.e., $10 \mathrm{M} \lambda$ correspond to a baseline of $16 \mathrm{~m}$ at $\lambda=1.6 \mu \mathrm{m}$. Baseline lengths range from $5 \mathrm{~m}$ to $38 \mathrm{~m}$.

(A color version of this figure is available in the online journal.)

Table 1

Observation Log

\begin{tabular}{lccc}
\hline \hline \multicolumn{1}{c}{ Date (UT) } & $\phi^{\mathrm{a}}$ & Configuration & Length $^{\mathrm{c}}(\mathrm{m})$ \\
\hline 2005 May 25 & 0.92 & A15-B15-C0 & 21 \\
2005 May 27 & 0.93 & A15-B15-C5 & 21 \\
2005 May 31 & 0.93 & A25-B15-C10 & 29 \\
2005 Jun 1 & 0.94 & A35-B15-C10 & 37 \\
\hline 2005 Oct 5, 6, & 0.25 & A5-B5-C0 & 7 \\
2005 Oct 8, 9 & 0.26 & A5-B15-C0 & 14 \\
2005 Oct 10, 11 & 0.26 & A15-B15-C0 & 21 \\
2005 Oct 12, 13 & 0.27 & A25-B15-C0 & 29 \\
\hline 2006 Mar 29, 31 & 0.68 & A15-B15-C0 & 21 \\
2006 Apr 2 & 0.69 & A5-B5-C0 & 7 \\
2006 Apr 7 & 0.70 & A30-B15-C0 & 33 \\
\hline 2006 May 11 & 0.78 & A15-B5-C10 & 15 \\
2006 May 12 & 0.78 & A15-B5-C0 & 15 \\
2006 May 13 & 0.79 & A15-B15-C0 & 21 \\
2006 May 14 & 0.79 & A30-B15-C0 & 33 \\
2006 May 15 & 0.79 & A35-B15-C21 & 38 \\
2006 May 16 & 0.79 & A35-B15-C25 & 38 \\
\hline
\end{tabular}

Notes.

a Stellar phase derived from AAVSO photometric observations.

${ }^{b}$ Interferometer configuration refers to the location, in meters, of telescopes A, $\mathrm{B}$, and $\mathrm{C}$ on the north/east, south/east, and north/east arms, respectively.

${ }^{c}$ Length of maximum projected baseline.

setup was implemented shortly before our last observation run, resulting in a data set featuring seven spectral channels covering the range $1.5 \mu \mathrm{m} \leqslant \lambda \leqslant 1.8 \mu \mathrm{m}$ (Pedretti et al. 2008). The science target observations are interleaved with identical observations of unresolved or partially resolved stars, used to calibrate the interferometer's instrumental response. The interleaved calibrator sources (listed in Table 2) were chosen in 

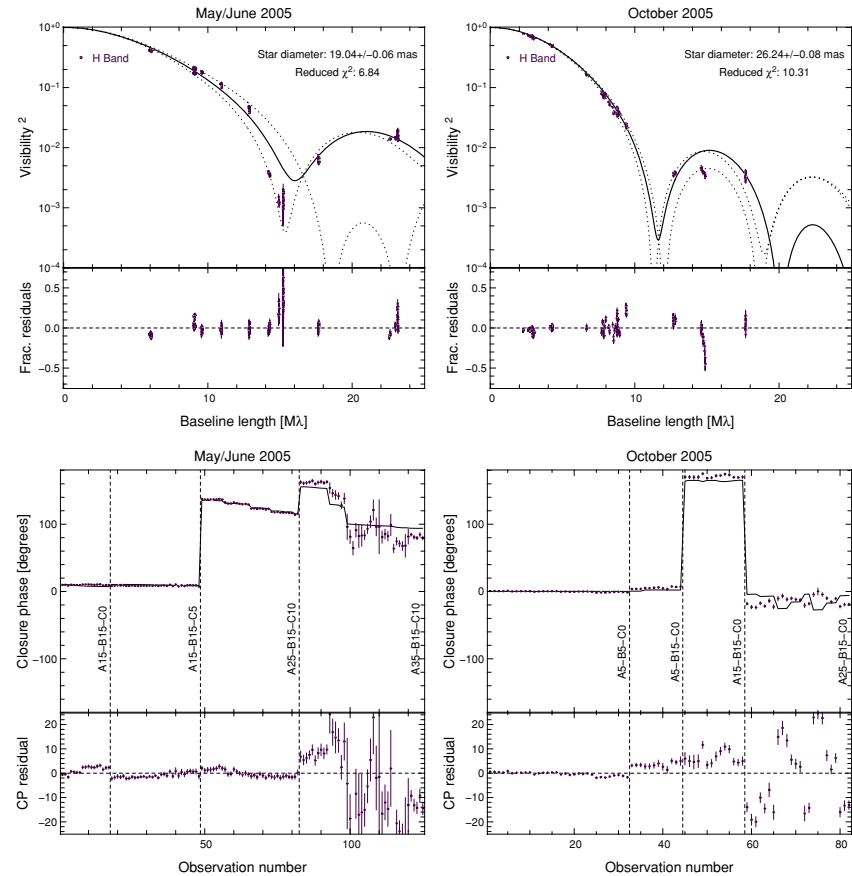

Figure 2. Squared visibilities (upper panels) and CPs (lower panels) at the two first epochs of observation. The curves correspond to the best fit of the model described in Figure 7. Three curves are plotted on the visibilities: the dashed curves are in the direction of the spot, and at $90^{\circ}$ from it. The solid curve is the visibility curve toward the longest baseline measured. The lower sub-panels show the residual errors.

Table 2

Calibrators

\begin{tabular}{lcc}
\hline \hline Calibrator & Spectral Type & UD Diameter \\
\hline HD 176670 & K2.5 III & $2.330 \pm 0.026$ \\
HD 180450 & M0 III & $2.770 \pm 0.032$ \\
HD 186619 & M0 IIIab & $2.190 \pm 0.025$ \\
HD 188149 & K4 III & $1.490 \pm 0.020$ \\
HD 197989 & K0 III & $4.440 \pm 0.048$ \\
\hline
\end{tabular}

two different catalogs: Bordé et al. (2002) and Mérand et al. (2006a)

Reduction of the IONIC3 data was carried out using custom software (Monnier et al. 2006), with exactly the same settings as those previously detailed in Lacour et al. (2008). The output of this reduction software is squared visibilities $\left(V^{2}\right)$ and CPs. Data are available upon request in the OIFITS format (Pauls et al. 2005). They are presented in the eight panels of Figures 2 and 3 (superimposed on the data are curves of the best fit of the model presented in Section 4). Analysis by eye of the $V^{2}$ (upper panels) shows a significant diameter variation between the different epochs. Also, at the longest baseline, the CPs are clearly different from 0 or $\pi$. This reveals the presence of an asymmetric brightness repartition, even though the complexity of CP estimators makes it hard to straightforwardly derive the level of asymmetry.

\section{REGULARIZED IMAGING}

A first step in our data analysis was to convey the squared visibilities and $\mathrm{CP}$ into spatial information. The imaging was performed by the MiRA reconstruction software. ${ }^{14}$ It stands for "Multi-aperture Image Reconstruction Algorithm" (Thiébaut

\footnotetext{
14 http://www-obs.univ-lyon1.fr/labo/perso/eric.thiebaut/mira.html
}
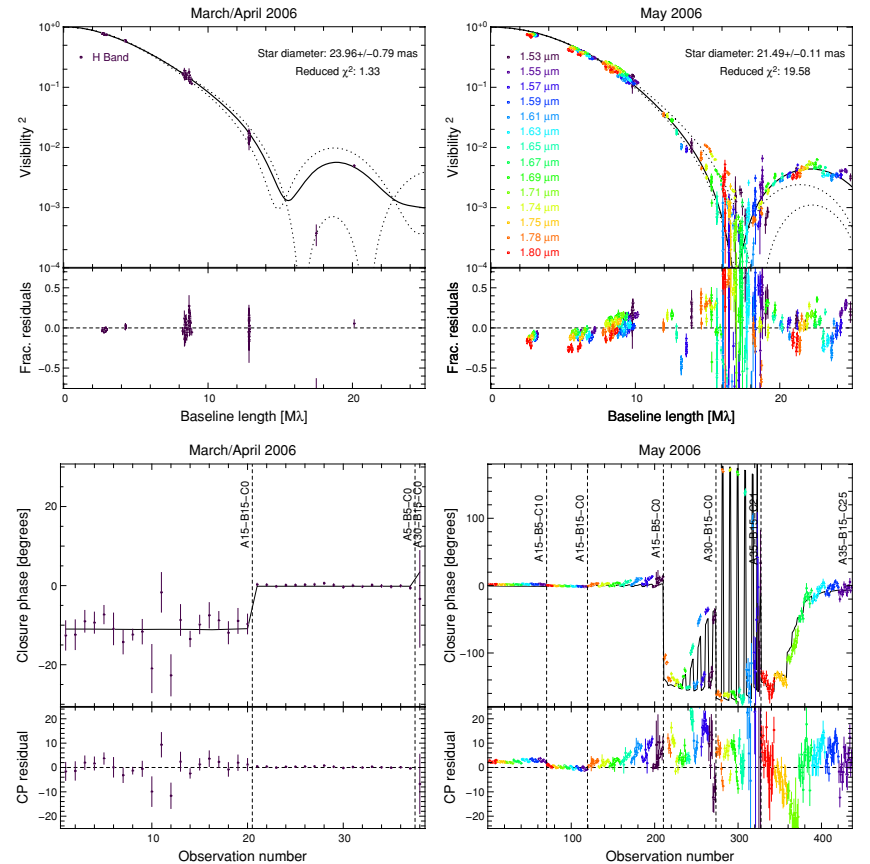

Figure 3. Squared visibilities (upper panels) and CPs (lower panels) at the two last epochs of observation. The curves correspond to the best fit of the model described in Figure 7. Three curves are present: the dashed visibility curves are in the direction of the spot, and at $90^{\circ}$ from it. The solid curve is the visibility curve toward the longest baseline measured. The lower sub-panels show the residual errors.

(A color version of this figure is available in the online journal.)

2008; Thiébaut et al. 2003). For more details, we refer the readers to previous image reconstruction work using this software (Lacour et al. 2008; Le Bouquin et al. 2009) as well as more theoretical descriptions (Le Besnerais et al. 2008; Thiébaut \& Giovannelli 2009).

Because the frequency coverage is far from being complete, the theoretical bijection between the frequency (Figure 1) and spatial domains is hard to achieve. It explains why the imaging algorithm requires a strong regularization term: the image is sought by minimizing a so-called cost function which is the sum of a regularization term plus data related terms. The data terms enforce the agreement of the model image with the different kinds of measured data (squared visibilities and phase closures). The interpolation of missing frequency coverage is performed by the regularization and by strict constraints such as the positivity (which plays the role of a floating support constraint) and normalization.

For $\chi$ Cygni, the regularization term was a $\chi^{2}$ minimization between the reconstructed image and a simple model of limbdarkened disk. Explicitly, the regularization term was $\alpha \sum_{k}\left(l_{k}-\right.$ $\left.m_{k}\right)^{2}$, where $\alpha$ is the relative weight of the regularization, $l_{k}$ is the intensity of the $k$ th pixel in the sought image, and $m_{k}$ is the flux of the $k$ th pixel given by the limb-darkening model. The $m_{k}$ is a model of the brightness distribution of a stellar disk whose parameters (diameter and power of the limb darkening) are beforehand adjusted on the squared visibilities. An advantage of this image reconstruction approach is that our prior favor radial symmetry. Hence this gives more strength to the relevancy of asymmetric features such as brighter regions in the restored images.

The results are presented in Figure 4. The four epochs are labeled by their stellar phase from upper left hand to lower right hand. The color scales of each image reconstruction are 


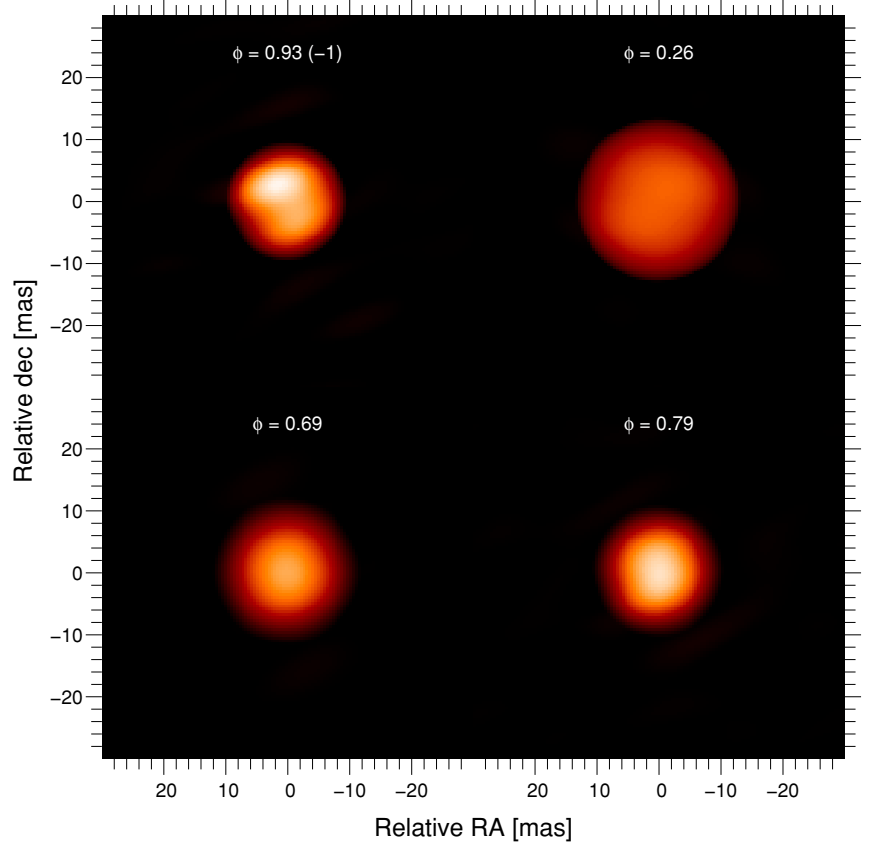

Figure 4. Regularized imaging of $\chi$ Cyg. The four epochs are labeled by their stellar phase, from upper left hand to lower right hand. The variation in diameter is striking with diameter changes of up to $40 \%$ between phases 0.93 and 0.26 . Changes in limb darkening are also present. Hot cells on the photosphere are apparent, interestingly with a higher contrast of the cells when the star is at a smaller diameter. The relative brightnesses are normalized according to $\mathrm{H}$-band magnitude from Table 5. The angular resolution of the IOTA interferometer is $10 \times 23$ mas $(1.65 \mu \mathrm{m})$. At $\chi$ Cygni's distance of $170 \mathrm{pc}, 5.9$ mas correspond to $1 \mathrm{AU}$ (Section 6.2.3).

(A color version of this figure is available in the online journal.)

the same. The total brightness of each epoch is normalized according to the bolometric flux estimated in Section 5.1.3.

The variation in diameter is eye striking. Radius (defined by a $10 \%$ of the maximum surface flux) ranges from $9.4(\phi=0.93)$ to 13.2 mas $(\phi=0.26)$. This means a $40 \%$ expansion of the photosphere between the two epochs. Curves in Figure 5 represent the radially averaged flux as a function of the radius. It is clear that, on top of diameter variations, changes in limb darkening are present, making it an important factor which has to be accounted for to determine precise photospheric radii.

\section{MODEL FITTING}

\subsection{Choosing the Right Geometrical Description}

Interferometric observation of Mira stars are traditionally interpreted by fitting simple brightness profiles, such as a uniform disk (UD) or a Gaussian disk (GD; Scholz 2003). Both fits showed inadequacy with our data. This is shown at the two first epochs of observation in Figure 6. At low frequency, the data are best fitted with a GD. At higher frequency, however, CPs $\pi$-shifted imply the presence of a second lobe, ruling out a Gaussian-type profile. With reduced $\chi^{2}$ well over several hundreds, these fits confirm the need for a more complex geometrical description.

Following an idea from Perrin et al. (1999), Mennesson et al. (2002) first introduced a two-component model for Mira stars. It was a disk limb darkened by a close warm molecular layer. This model did well to account for spectral measurements in the $K$ and $L$ bands. However, we noted some problems when using this model to fit our measurements. First, because this model is

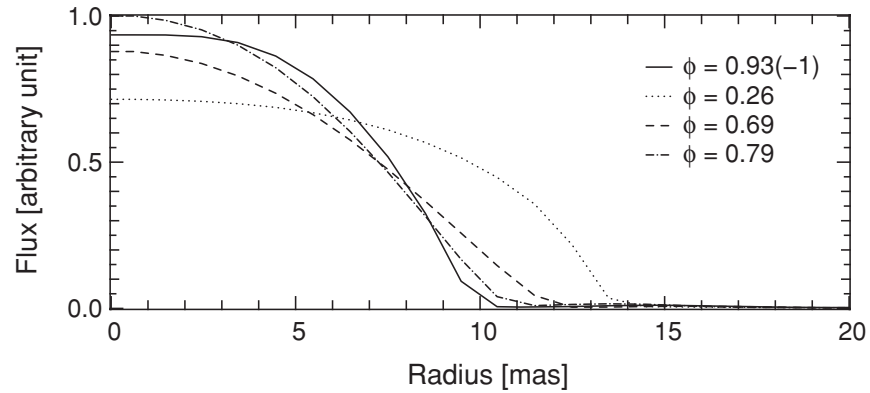

Figure 5. CLV at the four epochs of Figure 4. The flux is radially averaged. It shows a clear variation in the brightness distribution as a function of time.
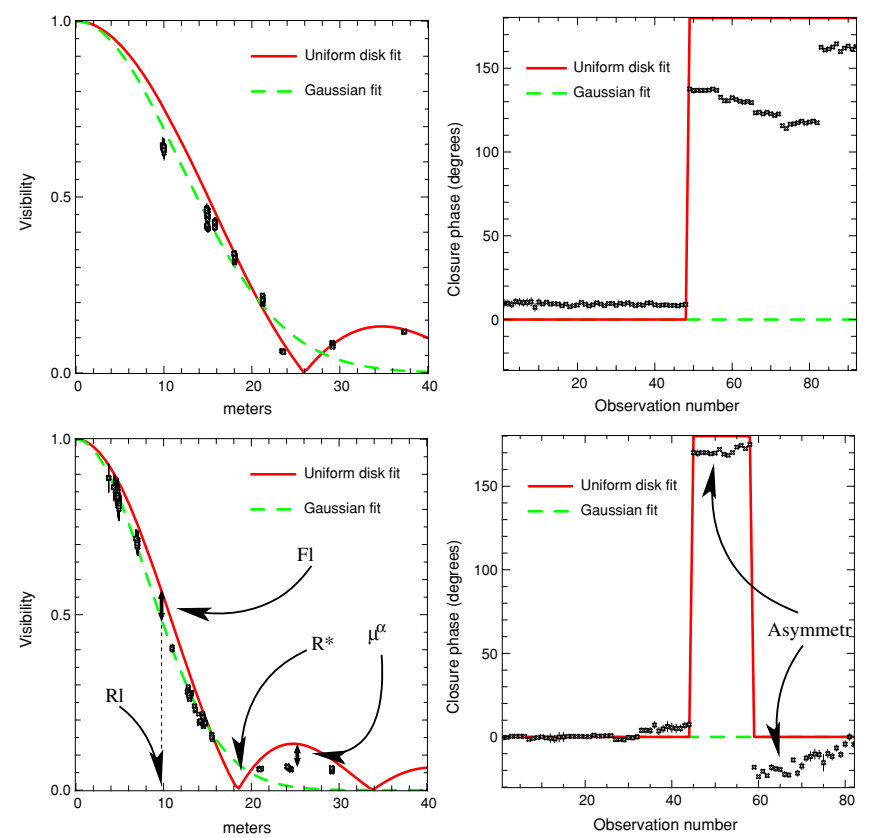

Figure 6. Data of 2005 May (upper panels) and 2005 October (lower panels). The best fits of a UD and a GD are plotted on top of the measurements. While the UD has trouble to fit the low frequency measurements, the GD is unable to account for the CP $\pi$-shift. In the two lower panels, the "zone of influence" of the parameters used in the model presented in Figure 7 is also summarized: the molecular layer (of flux $F_{l}$ and radius $R_{l}$ ) will impact the data at low frequency, the diameter of the star $\left(R_{\star}\right)$ will determine the position of the first null, and the limb darkening $\left(\mu^{\alpha}\right)$ will impact the higher frequency by modifying the height of the second lob. The asymmetry is clearly revealed by the CP.

(A color version of this figure is available in the online journal.)

spherically symmetric, it cannot account for CP measurements different from $0^{\circ}$ or $180^{\circ}$. Second, the physical parameters (temperature and optical depth) present a degeneracy which cannot be resolved without spectral information. We therefore decided to use a purely geometrical description of the brightness distribution. This model contains seven parameters describing the presence of a photosphere with a center-to-limb darkening, a circumstellar envelope, and a spot on the stellar surface (Figure 7).

The photosphere is modeled by a limb-darkened disk. The center-to-limb variation (CLV) is a simple, single-parameter, power law: $I(\mu) / I(1)=\mu^{\alpha}$ (Michelson \& Pease 1921; Hestroffer 1997); with $\mu=\cos (\theta)$, and $\theta$ is the angle between the line of sight and the radial vector from the center of the star. Compared to a more classical four-parameter law (as in Claret 2000), this law has the advantage of fitting various CLV shapes with a single parameter. The circumstellar envelope is modeled by an annular ring around the photosphere. This is a simple 


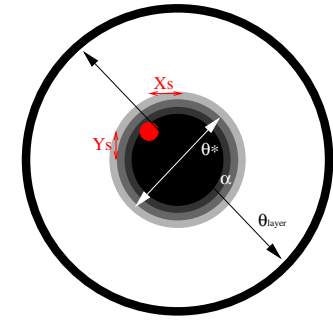

Figure 7. Model used in Section 4. It consists of a limb-darkened disk, a spot and a molecular layer represented by a ring. The free parameters are $\theta_{\star}, \theta_{\text {layer }}$ $\alpha, X_{s}, Y_{s}$, plus the brightness of the layer and the spot relative to the total flux. (A color version of this figure is available in the online journal.)

way to account for the existence of either (1) a warm molecular layer as proposed by Perrin et al. (2004) or (2) a molecular extension of the photosphere as modeled by Ireland et al. (2008). Finally, we needed a way to account for the asymmetry. We used the simplest model available to explain an asymmetry: a single point-like spot. The model allows either a "cool" (dark) or "hot" (bright) spot. There is no restriction on the position of the spot (it is allowed to be outside the photosphere).

To be sure that each one of these parameters are well constrained, it is crucial to know the influence they have on the visibility curve. The bottom panels of Figure 6 give rough estimations of the zone of influence of each parameter. At first order, the molecular layer is constrained by the low frequencies, the disk size by the first zero, the limb darkening by the height of the second lobe, and the asymmetry by the CP. Of course, to a lesser extent, all the parameters affect each other at various degrees.

\subsection{Fitting the Data}

An advantage of this model is that it consists in a sum of brightness distributions for which an analytical formula of the visibility function exists. The function of a power-law limbdarkened disk, of parameter $\alpha$ and diameter $\theta_{\star}$, writes

$$
V_{\star}\left(v_{r}\right)=\sum_{k \geqslant 0} \frac{\Gamma(\alpha / 2+2)}{\Gamma(\alpha / 2+k+2) \Gamma(k+1)}\left(\frac{-\left(\pi v_{r} \theta_{\star}\right)^{2}}{4}\right)^{k},
$$

where $v_{r}$ is the radial spatial frequency $\left(v_{r}=\sqrt{u^{2}+v^{2}}\right)$ and $\Gamma$ is the Euler function. The visibility function of an annular ring of diameter $\theta_{\text {layer }}$ is

$$
V_{\text {layer }}\left(v_{r}\right)=J_{0}\left(2 \pi \theta_{\text {layer }} v_{r}\right)
$$

where $J_{0}$ is the Bessel function of the first kind. Finally, the visibility function of a hot spot writes

$$
V_{\text {spot }}(u, v)=\exp \left(-2 i \pi\left(X_{\text {spot }} u+Y_{\text {spot }} v\right)\right),
$$

where the $X_{\text {spot }}$ and $Y_{\text {spot }}$ are the coordinates of the spot (respectively right ascension and declination) relative to the center of symmetry of the stellar surface. The visibility of the full model is the weighted sum of the three visibility functions, hence

$$
V(u, v)=F_{\star} V_{\star}\left(v_{r}\right)+F_{\text {layer }} V_{\text {layer }}\left(v_{r}\right)+F_{\text {spot }} V_{\text {spot }}(u, v) .
$$

$F_{\star}, F_{\text {layer }}$ and $F_{\text {spot }}$ are the relative fluxes of, respectively, the disk, the envelope, and the spot $\left(F_{\star}+F_{\text {layer }}+F_{\text {spot }}=1\right)$.

Closure phases are obtained by taking the argument of the product of three complex visibilities: $\arg \left(V\left(u_{1}, v_{1}\right) \cdot V\left(u_{2}, v_{2}\right)\right.$.
$\left.V\left(-u_{1}-u_{2},-v_{1}-v_{2}\right)\right)$. Squared visibilities are derived from the squared of the visibilities $\left(|V(u, v)|^{2}\right)$. Because $u$ and $v$ are wavelength dependent, accounting for the bandwidth smearing required one to average the squared visibilities before fitting them to the data. For the first three observation runs, they are averaged over the 1.59-1.69 $\mu \mathrm{m}$ bandpass. For the last run, the model was averaged over each channel bandpass $(\Delta \lambda=40 \mathrm{~nm})$. The model is supposed to be achromatic.

Thanks to the analytical expressions, the model can be swiftly fitted over a large range of parameter values. However, because the $\mathrm{CP}$ measurements are at the same time very precise $\left(\approx 1^{\circ}\right)$ and very sparse (there is only one phase measurements for three squared visibilities), the spot position can have multiple $\chi^{2}$ minima. To find the most likely set of parameters, we used the following strategy to achieve the global optimization of the $\chi^{2}$ : for a grid of given spot positions, we first map the $\chi^{2}$ minimized with respect to other parameters. Then we use the position which yields the best $\chi^{2}$ to initiate a local optimization with respect to all parameters by a Levenberg-Marquardt algorithm.

The upper panels of Figures 2 and 3 show the best fit of the model plotted on the squared visibilities (with a logarithmic scale). Since the asymmetry cannot be represented by a single radial visibility curve, we plotted three curves. The two dashed visibility curves are in the direction and at $90^{\circ}$ of the direction of the spot. The solid curve is the visibility curve toward the longest baseline measured. The difference between the model and the data points is plotted in the lower subpanels. Residual errors on the $V^{2}$ average around $1 \%$.

The lower panels of Figures 2 and 3 show the best fit of the model plotted on the CPs. Even though the point-like spot model is a very rough estimation of what the asymmetry could be, the general agreement between the model and the data confirms the validity of using such a simple representation. However, the results cannot exclude more complicated asymmetries, such as the presence of multiple spots or other heterogeneities. For the October observations, for example, small variations in the $\mathrm{CP}$ are not well matched by our model, hinting for a more complex repartition of the asymmetry.

We noted that the minimum reduced $\chi^{2}$ can be quite different from 1, ranging from a value of 1.3 (2006 March) to 19.6 (2006 May). Lower $\chi^{2}$ can be obtained by using more complex models, like fitting two spots instead of one, or adjusting a chromatic limb darkening to the 2006 May data. However, the multiple parameters in such a case were too badly constrained to allow good determination. In the end, we decided to stay with our most simple model which gives a good compromise between fitting the data well and a reasonable number of free parameters.

\subsection{Parametric Imaging}

The results and error bars of the fits can be found in Table 3. From these values, we can retrieve a brightness distribution of the object, i.e., an image. The resulting images are presented in Figure 8, and should be compared to the regularized images of Figure 4. Concordance is quite convincing, with a remarkable reproductivity in terms of diameter and position of the hot spots. The main difference is the presence of the molecular layer, situated around 0.5 stellar radius above the photosphere. The faintness of the layer (a faintness expected within our wavelength range of observation) may explain why the regularization algorithm did not image it. This on top of the fact that the regularization term does favor an empty environment. 
Table 3

Best-fit Parameters

\begin{tabular}{lcccc}
\hline \hline Parameter & $\begin{array}{c}2005 \text { May/June } \\
(\phi=0.93)\end{array}$ & $\begin{array}{c}2005 \text { October } \\
(\phi=0.26)\end{array}$ & $\begin{array}{c}\text { 2006 March/April } \\
(\phi=0.69)\end{array}$ & $\begin{array}{c}2006 \text { May } \\
(\phi=0.79)\end{array}$ \\
\hline$\theta_{\star}(\mathrm{mas})$ & $19.04 \pm 0.09$ & $26.25 \pm 0.08$ & $23.97 \pm 0.80$ & $21.49 \pm 0.11$ \\
$\mathrm{LD}[\alpha]$ & $1.34 \pm 0.05$ & $1.08 \pm 0.03$ & $2.54 \pm 0.39$ & $2.42 \pm 0.05$ \\
$\theta_{\text {layer }}(\mathrm{mas})$ & $31.83 \pm 0.15$ & $40.75 \pm 0.37$ & $35.48 \pm 0.40$ & $27.35 \pm 0.13$ \\
$F_{\text {layer }} / F_{\text {total }}(\%)$ & $6.5 \pm 0.2$ & $4.7 \pm 0.2$ & $8.8 \pm 0.3$ & $8.13 \pm 0.2$ \\
$X_{\text {spot }}(\mathrm{mas})$ & $5.22 \pm 0.05$ & $-8.92 \pm 0.39$ & $2.22 \pm 0.42$ & $3.21 \pm 0.20$ \\
$Y_{\text {spot }}(\mathrm{mas})$ & $2.97 \pm 0.05$ & $2.96 \pm 0.10$ & $-4.24 \pm 0.34$ & $-6.70 \pm 0.09$ \\
$F_{\text {spot }} / F_{\text {total }}(\%)$ & $5.9 \pm 0.1$ & $1.7 \pm 0.1$ & $3.7 \pm 0.3$ & $1.7 \pm 0.1$ \\
\hline Reduced $\chi^{2}$ & 6.8 & 10.3 & 1.33 & 19.6 \\
\hline
\end{tabular}

Table 4

Model-dependent Diameter Measurements

\begin{tabular}{|c|c|c|c|c|c|c|}
\hline \multirow{2}{*}{$\begin{array}{c}\text { Model } \\
\text { Parameter }\end{array}$} & \multicolumn{3}{|c|}{2005 May $(\phi=0.93)$} & \multicolumn{3}{|c|}{2005 October $(\phi=0.26)$} \\
\hline & UD & UD + Layer + Spot & LD + Layer + Spot & UD & UD + Layer + Spot & LD + Layer + Spot \\
\hline$\overline{\theta_{\star} \text { (mas) }}$ & $16.24 \pm 0.07$ & $14.99 \pm 0.07$ & $19.04 \pm 0.09$ & $22.99 \pm 0.11$ & $20.90 \pm 0.12$ & $26.25 \pm 0.08$ \\
\hline$\alpha$ & $\ldots$ & $\ldots$ & $1.35 \pm 0.05$ & $\ldots$ & $\ldots$ & $1.08 \pm 0.03$ \\
\hline$\theta_{\text {layer }}$ (mas) & $\ldots$ & $21.76 \pm 0.27$ & $31.85 \pm 0.15$ & $\ldots$ & $26.76 \pm 0.24$ & $40.75 \pm 0.37$ \\
\hline$F_{\text {layer }} / F_{\text {TOTAL }}(\%)$ & $\ldots$ & $10.9 \pm 0.3$ & $6.5 \pm 0.2$ & $\ldots$ & $15.1 \pm 0.6$ & $4.7 \pm 0.2$ \\
\hline$X_{\text {spot }}(\mathrm{mas})$ & $\ldots$ & $5.47 \pm 0.14$ & $5.22 \pm 0.05$ & $\cdots$ & $-8.4 \pm 0.78$ & $-8.92 \pm 0.39$ \\
\hline$Y_{\text {spot }}(\mathrm{mas})$ & $\ldots$ & $3.26 \pm 0.11$ & $2.97 \pm 0.05$ & $\ldots$ & $2.15 \pm 0.17$ & $2.96 \pm 0.10$ \\
\hline$F_{\text {spot }} / F_{\text {TOTAL }}(\%)$ & $\ldots$ & $5.9 \pm 0.2$ & $5.9 \pm 0.1$ & $\ldots$ & $1.2 \pm 0.1$ & $1.7 \pm 0.1$ \\
\hline Reduced $\chi^{2}$ & 550 & 29 & 6 & 536 & 14 & 10 \\
\hline
\end{tabular}

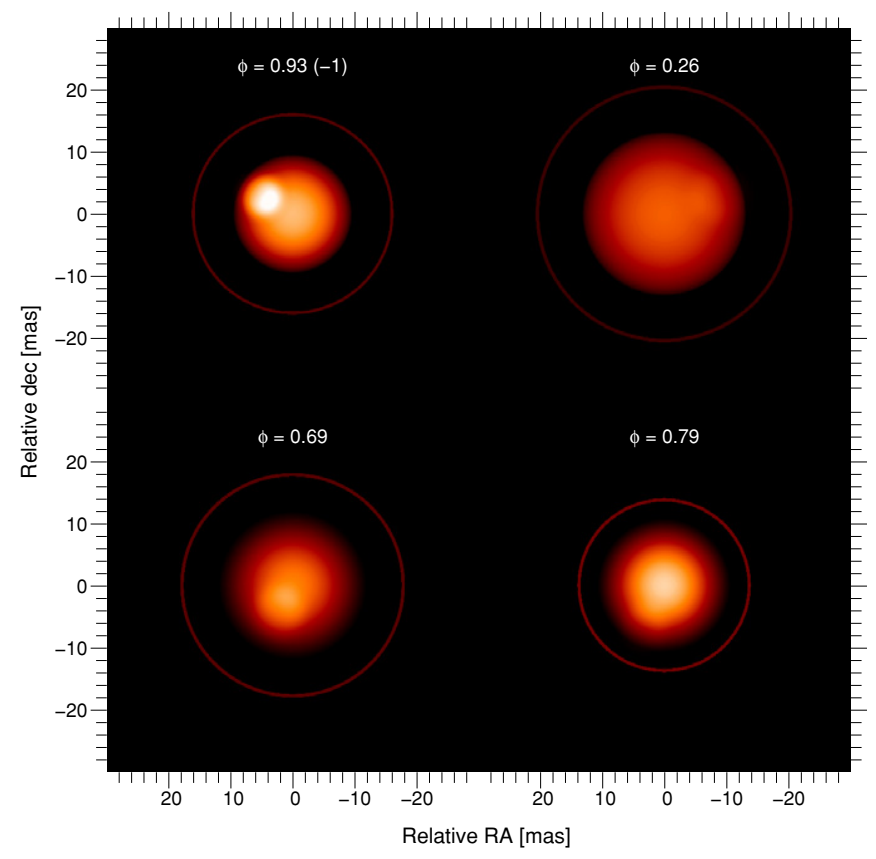

Figure 8. Parametric imaging. The parametric model is shown in Figure 7. The values used are those reported in Table 3. Apparency of the spot was chosen to be the fifth of the stellar diameter, below the resolution power of the interferometer. The relative brightnesses are normalized according to the H-band magnitude from Table 5. At $\chi$ Cygni's distance of $170 \mathrm{pc}, 5.9$ mas corresponds to $1 \mathrm{AU}$ (Section 6.2.3).

(A color version of this figure is available in the online journal.)

\section{LINKING SPATIAL TO PHYSICAL PARAMETERS}

\subsection{Stellar Diameter}

\subsubsection{Rosseland Radius}

In the case of an extended-atmosphere star such as Mira variables, a sensible definition of the radius as to be agreed upon. A quantity mostly used in Mira modeling is the Rosseland radius. This is an optical depth radius $\left(R_{\text {Ross }}=r\right.$ where $\tau_{\text {Ross }}=1$ ) and unfortunately not an observable quantity. In the case of the solar disk, the photospheric radius of the Sun is defined by the position of the CLV inflection point.

The diameter values stated in Table 3 correspond to the furthest emission point of the photosphere, molecular layer notwithstanding. The problem of this definition of diameter is that it is highly model dependent. Such influence is emphasized in Table 4. The table compares the results of three different models fitted to the data set of two epochs. It shows how profound the difference on diameter measurements can be depending on the model, well outside the range of error bars. A solution could have been to consider only the UD radius, and to use tables (for example in Davis et al. 2000) to derive the Rosseland radius from simulations (Ireland et al. 2004a, 2004b). However, this technique poses the problem of fitting a UD on a data set which is not compatible with it: the bias on the UD diameters depends on the Fourier coverage (Paladini et al. 2009).

However, a noticeable advantage of this work is the good coverage of spatial frequencies at all epochs. Our model, which covers a large range of CLV possibilities, allows us to disentangle the main features of the stars (layer, spot). Simulations of pure-continuum brightness profiles have steep flanks which mark the position of continuum-forming layers (Scholz \& Takeda 1987). We are confident that the steep flank observed on the fitted model marks, as rightly as possible, the limit of the Rosseland radius.

\subsubsection{Comparison with other Interferometric Observations}

Numerous $\chi$ Cyg diameter measurements are present in the literature, but the multiple techniques of determination makes comparison difficult. The first near-infrared interferometric observations of $\chi$ Cyg were obtained by Young et al. (2000) using the COAST instrument. In the $1.3 \mu \mathrm{m}$ continuum bandpass, they obtained a Gaussian FWHM of $13.9 \pm 0.8$ mas at $\phi=0.83$. 
Table 5

Bolometric Flux

\begin{tabular}{lccrr}
\hline \hline \multicolumn{1}{c}{$\phi$} & 0.96 & 0.26 & 0.69 & \multicolumn{1}{c}{0.79} \\
\multicolumn{1}{c}{$\mathrm{JD}$} & 2453518 & 2453653 & 2453826 & \multicolumn{1}{c}{2453869} \\
\hline$J(\mathrm{mag})^{\mathrm{a}}$ & $0.00 \pm 0.15$ & $-0.46 \pm 0.15$ & $0.15 \pm 0.15$ & $0.07 \pm 0.15$ \\
$H(\mathrm{mag})^{\mathrm{a}}$ & $-1.00 \pm 0.15$ & $-1.65 \pm 0.15$ & $-1.05 \pm 0.15$ & $-1.01 \pm 0.15$ \\
$K(\mathrm{mag})^{\mathrm{a}}$ & $-1.65 \pm 0.15$ & $-2.24 \pm 0.15$ & $-1.73 \pm 0.15$ & $-1.65 \pm 0.15$ \\
$L(\mathrm{mag})^{\mathrm{a}}$ & $-2.50 \pm 0.15$ & $-2.84 \pm 0.15$ & $-2.51 \pm 0.15$ & $-2.48 \pm 0.15$ \\
$F_{\mathrm{Bol}}$ & & & & $6.74 \pm 0.36$ \\
$\left(10^{-13} \mathrm{~W} \mathrm{~cm}^{-2}\right)$ & $6.83 \pm 0.38$ & $10.15 \pm 0.57$ & $6.80 \pm 0.35$ & 6.75 \\
\hline
\end{tabular}

Note. ${ }^{\text {a }}$ Magnitudes obtained from Whitelock et al. (2000).

Table 6

Time-Dependent Parameters of $\chi$ Cyg

\begin{tabular}{lcccc}
\hline \hline \multicolumn{1}{c}{$\phi$} & 2005 May/June & 2005 October & 2006 March/April & 2006 May \\
& 0.93 & 0.26 & 0.69 & 0.79 \\
\hline$\theta_{\star}(\mathrm{mas})$ & $19.04 \pm 0.09$ & $26.25 \pm 0.08$ & $23.97 \pm 0.80$ & $21.49 \pm 0.11$ \\
$R_{\star} / R_{\odot}{ }^{\mathrm{a}}$ & $348 \pm 94$ & $480 \pm 130$ & $439 \pm 119$ & $393 \pm 106$ \\
$T_{\star}(\mathrm{K})$ & $2742 \pm 45$ & $2578 \pm 40$ & $2441 \pm 72$ & $2572 \pm 41$ \\
\hline$\theta_{\text {layer }}(\mathrm{mas})$ & $31.83 \pm 0.15$ & $40.75 \pm 0.37$ & $35.48 \pm 0.40$ & $27.35 \pm 0.13$ \\
$R_{\text {layer }} / R_{\star}$ & $1.67 \pm 0.02$ & $1.55 \pm 0.02$ & $1.48 \pm 0.07$ & $1.27 \pm 0.01$ \\
$T_{\text {layer }}(\mathrm{K})$ & $1821 \pm 29$ & $1795 \pm 28$ & $1747 \pm 52$ & $2032 \pm 32$ \\
$\tau_{\text {layer }}$ & $0.042 \pm 0.002$ & $0.032 \pm 0.002$ & $0.067 \pm 0.007$ & $0.074 \pm 0.002$ \\
\hline$p^{\mathrm{b}}$ & $1.22 \pm 0.01$ & $1.26 \pm 0.01$ & $1.22 \pm 0.02$ & $1.27 \pm 0.01$ \\
\hline
\end{tabular}

Notes.

a Assuming a parallax of $5.9 \pm 1.5$ mas (Section 6.2.3).

b Projection factor (Section 6.2.2).

The $44 \%$ discrepancy with our diameter of $19.04 \pm 0.09$ mas at $\phi=0.93$ is difficult to explain by the difference of phase only. However it can be explained by the study of Hofmann et al. (1998). In their paper, they showed that the ratio between continuum radius and Gauss radius should be around 0.6 , a value close to what is observed here. Perrin et al. (2004) used the beam combiner FLUOR on IOTA to obtain narrow band measurements in the $K$ band. They fitted a more complete model including a UD and a molecular layer with a wavelength-dependent optical depth. They found an apparent stellar diameter of $21.10 \pm 0.02$ mas at $\phi=0.24$. This value is compatible with our measurements of $20.90 \pm 0.12$ mas obtained by fitting a model without limb darkening, but not with the diameter of $26.25 \pm 0.08$ mas measured when accounting for the CLV shape. So the discrepancy is logically explained by the fact that they did not account for the limb darkening.

Another important diameter value still to be mentioned is the one obtained by Weiner et al. (2003) using the ISI heterodyne interferometer. They reported a diameter value of $39.38 \pm 4.02$ mas at $\phi=0.51$ in the $11 \mu \mathrm{m}$ continuum bandpass. Even though the phase of observation is different from ours, this value is far from being compatible. A possible interpretation could be the one proposed by Ohnaka (2004), suggesting the presence of a warm, spectroscopically "hidden," $\mathrm{H}_{2} \mathrm{O}$ molecular layer. It is interesting to note that the $11 \mu \mathrm{m}$ stellar diameter measurements match our measurements of the position of the molecular layer, something already noticed by Perrin et al. (2004).

Concerning the limb darkening, this paper offers the first measurements on a Mira star. But other observations exist on other types of stars. Mérand et al. (2006b) reported good fits with $\alpha=0.16$ toward Polaris and $\delta$ Cepheid, two variable Cepheids. Wittkowski et al. (2006) and Lacour et al. (2008) observed
$\mathrm{M}$ giant stars and obtained respectively $\alpha=0.24 \pm 0.03$ for Menkar and $\alpha=0.258 \pm 0.003$ for Arcturus. In comparison, $\chi$ Cygni's $\alpha$ values (greater than 1) look large. However, one has to consider the size and pulsation of the photosphere of Mira variables. In that respect, our values are compatible with the CLV simulations presented in Jacob \& Scholz (2002).

\subsubsection{Effective Temperature}

The effective temperature of a star depends upon its angular diameter and its bolometric flux,

$$
\sigma \cdot T_{\star}^{4}=\frac{4}{\theta_{\star}^{2}} \cdot F_{\mathrm{Bol}},
$$

where $\sigma$ is the Stefan-Boltzmann radiation constant, and $F_{\mathrm{Bol}}$ is the observed flux, integrated over all wavelengths. Whitelock et al. (2000) reported $J, H, K$, and $L$ band observations of the star at several phases. Thus, a phase-dependent bolometric flux can be estimated by fitting a black body distribution on the reported magnitudes. In Table 5, we present the photometric estimations as well as the bolometric flux at the epochs of observations. Applying Equation (7), we deduced the effective temperatures (reported in Table 6). They are interesting in the sense that they are much cooler than what was deduced in Perrin et al. (2004) and are consistent with an M8 spectral type (Perrin et al. 1998).

\subsubsection{A Time-variable View of $\chi$ Cygni}

Figure 9 represents the flux, radius, and temperature as a function of time. We fitted a sinusoidal model to the data. Bestfit values are reported in Table 7. Linear radius is equal to 12.1 mas and the amplitude of the pulsation is 5.2 mas (43\%). Minimum diameter happens at $\phi=0.94 \pm 0.01$. As expected, 

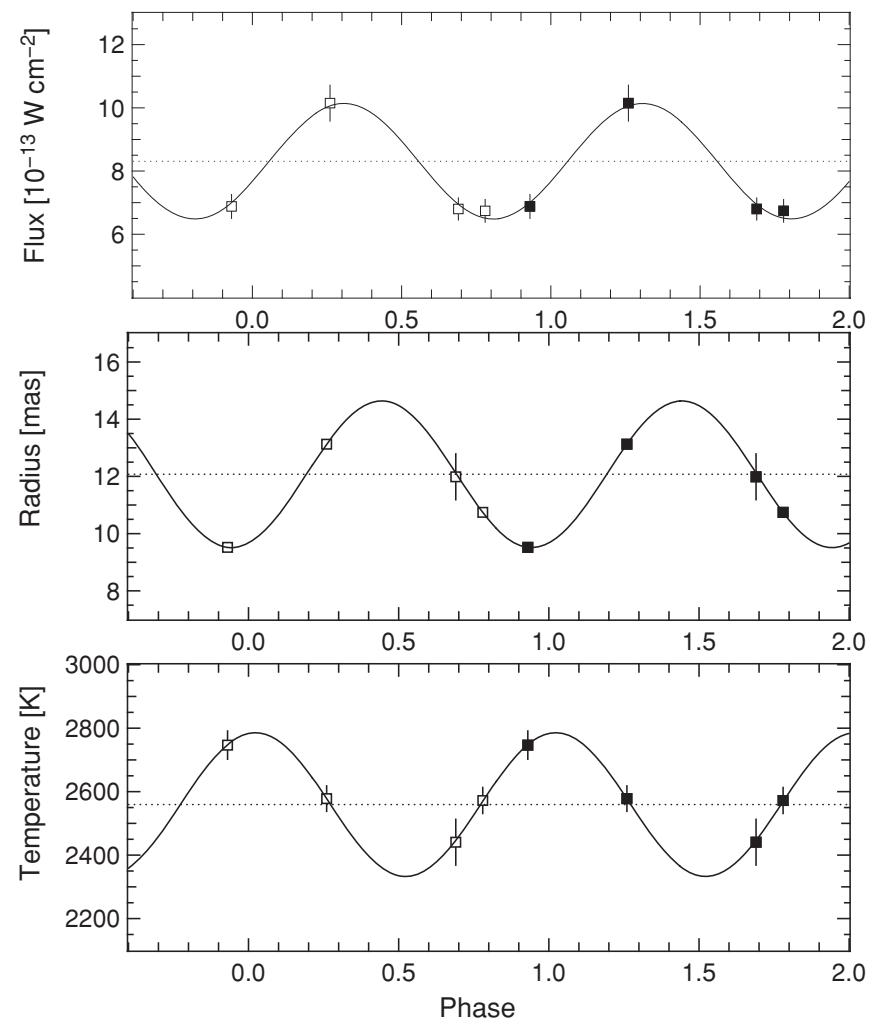

Figure 9. Temporal evolution of bolometric flux, radius and temperature of the photosphere of $\chi$ Cyg. Values (Table 6 ) are plotted twice with +1 phase shift (white and black dots). A sinusoidal is fitted to the data points, giving a linear radius of 12.1 mas and an average effective temperature of $2560 \mathrm{~K}$ (Table 7). The temperature was derived according to $T_{\text {eff }}=\left(4 \sigma F_{\mathrm{Bol}} / \theta_{\star}^{2}\right)^{1 / 4}$. It follows a periodic variation phase shifted by 0.58 compared to the radius, i.e., almost opposed to the dilatation of the photosphere.

Table 7

Sinusoidal Fit ${ }^{\text {a }}$

\begin{tabular}{lrcc}
\hline \hline \multicolumn{1}{c}{ Parameter } & \multicolumn{1}{c}{$a$} & \multicolumn{1}{c}{$b$} & $\phi_{0}$ \\
\hline$F_{\text {Bol }}\left(10^{-13} \mathrm{~W} \mathrm{~cm}^{-2}\right)$ & $8.3 \pm 0.3$ & $1.8 \pm 0.4$ & $0.30 \pm 0.03$ \\
Diameter (mas) & $24.2 \pm 0.1$ & $5.1 \pm 0.1$ & $0.44 \pm 0.01$ \\
Temperature $(\mathrm{K})$ & $2560 \pm 30$ & $226 \pm 56$ & $0.02 \pm 0.02$ \\
\hline
\end{tabular}

Note. ${ }^{\text {a }}$ Sinusoidal defined by $a+b \sin \left(2 \pi\left(\phi-\phi_{0}\right)\right)$.

the temperature $T$ is anti-correlated with the diameter, with a slight lag of $8 \%$ of the period $(\approx 30$ days). This anti-correlation, as well as a similar lag, is also observed on Cepheids stars (Andrievsky et al. 2005).

On the other hand, the bolometric flux is mostly correlated with the diameter, an indication that the variation in the bolometric flux is dominated by the variation of the size of the object. The temperature plays a minor role on the bolometric flux, but making it phase shifted by 0.14 in advance of phase compared to the radius. It is interesting to note that the visual magnitude, strongly affected by the molecular environment, is not in phase with the bolometric flux, but instead is in phase with the temperature.

There are not many time-variable radius and temperature figures in the literature. Young et al. (2000) observed a variation with a maximum diameter at phase 0.6 , but observations were done at a shorter wavelength $(905 \mathrm{~nm})$ which is affected by $\mathrm{TiO}$ absorption/emission. No noticeable diameter variation was observed in the less affected $1.3 \mu \mathrm{m}$ bandpass. On the other hand, Thompson et al. (2002) observed a clear variation in diameter in the near-infrared ( $\mathrm{K}$ band) toward the Mira star R Tri Mira. They observed a 10\% decrease in the UD diameter between phase 0.77 and 0.91 . It agrees with the variation in radius observed here on $\chi$ Cyg, but unfortunately the time coverage on $\mathrm{R}$ Tri does not allow a more through-full comparison. Noteworthy is the work done by Woodruff et al. (2008): they derived time-dependent UD diameter for eight Mira variables, including $\chi$ Cygni. Minimum diameter is observed at phase $\approx 0.7$, a value inconsistent with our work. This could be explained by the fact that they fitted UD diameters which do not account for the molecular environment of the star.

Least, a plot similar to the Figure 9 is present in the $\mathrm{PhD}$ Thesis of Strecker (1973). He used bolometric flux and $3.5 \mu \mathrm{m}$ observations of $\chi$ Cyg to deduce a time-variable temperature and stellar diameter. In terms of variations, his results match our data set well, with a minimum diameter around 0.9 and a minimum temperature at $\phi=0.5$. However, compared with our work, he overestimated the size of the star by a factor $1.5(\approx 36 \mathrm{mas})$ and underestimated its effective temperature $(\approx 2000 \mathrm{~K})$.

\subsection{Temperature and Opacity of the Molecular Layer}

The highly chromatic brightness of the layer observed by Perrin et al. (2004) immediately suggested the presence of molecules, mainly $\mathrm{H}_{2} \mathrm{O}$ and $\mathrm{CO}$. Because of its molecular nature, it is extremely difficult to disentangle the relative effect of temperature and opacity on the brightness.

To derive a first-order estimation of the temperature, we used the assumption of a gray atmosphere. Hence, from Reid \& Menten (1997), the temperature writes

$$
T_{\text {layer }}^{4}=T_{\star}^{4}\left(1-\sqrt{1-\left(\theta_{\star} / \theta_{\text {layer }}\right)^{2}}\right) .
$$

From this first-order estimation of the temperature, we can also deduce the optical depth of the molecular layer $\left(\tau_{\text {layer }}\right)$ by using the flux conservation relation:

$$
\frac{F_{\text {layer }}}{F_{\star}}=\frac{B\left(\lambda, T_{\text {layer }}\right)}{B\left(\lambda, T_{\star}\right)} \cdot \frac{\theta_{\text {layer }}^{2}}{\theta_{\star}^{2}} \cdot \frac{1-\exp \left(-\tau_{\text {layer }}\right)}{\exp \left(-\tau_{\text {layer }}\right)},
$$

where $B(\lambda, T)$ is the Planck function and $\lambda$ is the wavelength. Hence,

$$
\tau_{\text {layer }}=\ln \left(1+\frac{F_{\text {layer }}}{F_{\star}} \cdot \frac{B\left(\lambda, T_{\star}\right) \theta_{\star}^{2}}{B\left(\lambda, T_{\text {layer }}\right) \theta_{\text {layer }}^{2}}\right) .
$$

The temperature and optical depth are reported in Table 6. With an effective temperature ranging from $1750 \mathrm{~K}$ to $2000 \mathrm{~K}$, the layer is significantly cooler than the excitation state of $\mathrm{CO}$ $(\Delta v=3)$ molecules as observed by Hinkle et al. (1982). On the other hand, the temperature agrees well with multi-wavelength interferometric observation from Perrin et al. (2004). The optical depth of the molecular layer is very low $(\tau<0.1)$, something we expected in the $H$ band (and already reported on other Mira stars, as toward S Ori in Wittkowski et al. 2008).

\section{DERIVING MASS AND DISTANCE FROM THE KINEMATICS OF THE ATMOSPHERE}

\subsection{On the Existence of a Molecular Layer}

There is a strong debate about the physical nature of the warm molecular layer as seen by optical interferometry. Precise interferometric values (Perrin et al. 2004) and image reconstruction 
work (Le Bouquin et al. 2009) tend to see a shell-like structure. Simulations (Scholz 2003) would favor a continuum emission from the photosphere up to a certain height in the atmosphere. This work neither pretend nor wish to resolve this issue. In the previous section, we chose to use the shell-like layer approach to understand our data, knowing we are lacking the resolving power to convincingly distinguish between the two. From a geometrical point of view, the only difference between the two approaches only lies in the width of the layer, which is negligible in a shell-like representation.

Whatever the width of the molecular layer, clues on its kinematics are offered by dynamical modeling of the pulsating atmosphere (Bertschinger \& Chevalier 1985; Bowen 1988; Bessell et al. 1996). Specifically, Bertschinger \& Chevalier (1985) showed that the atmosphere should be periodically criss-crossed by supersonic shocks. They computed a postshock density 59 times the density of the pre-shock, forming a zone in which rapid cooling would allow high nucleation rates (Willson 2000). The material is then inwardly accelerated by the gravitational field, passing the sonic point closely below the shock front. At this time, pressure forces become unimportant, making the rest of the trajectory ballistic-like. This theory nicely explains how warm molecules-and eventually grains-could be formed so low within the atmosphere of the star.

If this model holds, the formation rates would decide the width of the molecular layer: density and temperature would define a spatially delimited zone in which molecules would happen to be more concentrated than in other atmospheric areas.

\subsection{A Modified Baade-Wesselink Method to Derive the Distance}

\subsubsection{The $C O(\Delta v=3)$ Absorption Features}

The Baade (1926) and Wesselink (1946) method consisted originally in deriving the absolute diameter and distance of a star by means of photometry and spectroscopy. The interferometric Baade-Wesselink method (applied to Cepheids in Kervella et al. 2004) differs by using a direct angular diameter measurement instead of photometry. We propose here to adapt this method to Mira stars, using the angular acceleration of the molecular shell. Concretely, the method allows deriving the distance by the relation:

$$
\text { parallax (mas) }=\frac{1}{p} \cdot \frac{g_{\text {angular }}\left(\mathrm{mas} / \mathrm{s}^{2}\right)}{g_{\text {radial velocity }}\left(\mathrm{AU} / \mathrm{s}^{2}\right)},
$$

where $p$ is a projection factor, $g_{\text {angular }}$ is the geometric acceleration observed by interferometry, and $g_{\text {radial velocity }}$ is the absolute acceleration observed by radial velocity. An alternative method could have been to use the speed instead of the acceleration (first derivative of size instead of second derivative). The advantage of using the acceleration is to avoid a bias due to uncertainties in the local stellar velocity.

Radial velocity of the molecular shell can be obtained through the $\mathrm{CO}$ second overtone vibration-rotation transitions $(\Delta v=3)$, observable by spectroscopy around $1.6 \mu \mathrm{m}$ (Hinkle et al. 1982). The high vibrational energy of these molecules is the characteristic of a warm environment ( $\mathrm{CO}$ molecules in lower vibrational states are also observed but probe a cooler environment farther away from the photosphere). These excited molecules follow a roughly similar path on most of Mira stars, tightly correlated with the visual phase: they are created around maximum visual brightness, steadily accelerated toward the star, and destroyed at the following stellar maximum (Hinkle
1978, 1995; Hinkle et al. 1984, 1997; Lebzelter et al. 2005). The excitation temperature is also correlated with the phase, showing a neat exponential cooling of the falling material. Line doubling is sometimes observed when the rotationally hot molecules form before complete dissociation of the less excited ones. This behavior is making considerable sense in the light of a Bertschinger \& Chevalier (1985) scenario summarized in the previous section.

One should be aware that using the Baade-Wesselink method in these conditions makes use of two strong hypotheses:

1. The molecular layer expansion is radial.

2. The molecular layer as seen by interferometry is the one from which originates the $\mathrm{CO}(\Delta v=3)$ absorption.

The radial nature of the $\mathrm{CO}$ displacement is a likely assumption because of the cycle to cycle repeatability as observed by Hinkle et al. (1982). The question of the concomitance of the molecular layer with the CO $(\Delta v=3)$ molecule is sensible, especially with respect to the unknown width of the molecular layer. Regarding the effective temperature, the depth of the absorption lines reported by Hinkle et al. (1982) match $1700 \mathrm{~K}$, close to the temperature of the layer reported in Table 6 . On the other hand, the excitation temperature is higher, but the exponential decrease from $4400 \mathrm{~K}$ to $2200 \mathrm{~K}$ observed by Hinkle et al. (1982) is hinting that excitation and effective temperatures are unlikely to be in equilibrium.

In the near future, we expect high spectral resolution interferometers to lift this uncertainty by probing the apparent angular diameter of the star directly within the absorption line.

\subsubsection{The Projection Factor}

The projection factor is one of the limiting parameters of the Baade-Wesselink methods. However, in the case of a Mira star imaged by interferometry, we can use the brightness distribution of the photosphere and combine it with the photosphere and molecular layer angular diameter. Assuming a shell of zero thickness, the projection factor writes

$$
p=\frac{\int_{0}^{R_{\star}} 2 \pi r \mu_{\star}^{\alpha} d r}{\int_{0}^{R_{\star}} 2 \pi r \mu_{\star}^{\alpha} \mu_{\text {layer }} d r},
$$

where $\mu_{\star}$ and $\mu_{\text {layer }}$ are the projected radii, i.e., $\mu_{\star}=(1-$ $\left.\left(r / R_{\star}\right)^{2}\right)^{1 / 2}$ and $\mu_{\text {layer }}=\left(1-\left(r / R_{\text {layer }}\right)^{2}\right)^{1 / 2}$. Using this formula and the numbers stated in Table 6 , the projection factor at the four phases of observations range from 1.22 to 1.27 . In the following section, we will use the projection factor $p=1.245 \pm 0.025$.

\subsection{3. $\chi$ Cygni Parallax}

In the upper panel of Figure 10 radial velocity data from Hinkle et al. (1982) are plotted. For convenience, the data have been folded in phase, and shifted by $9.6 \mathrm{~km} \mathrm{~s}^{-1}$. This velocity shift comes from an estimation of the local stellar velocity from CO measurements by Wannier et al. (1990), but is not relevant to derive the acceleration. We performed a linear fit over the period $(0<\phi<0.8)$ and derived a mean acceleration $g_{\text {radial velocity }}=-(1.10 \pm 0.04) \mathrm{mm} \mathrm{s}^{-2}$. Simultaneously, we fitted a parabola of constant inward acceleration over the position of the molecular layer (lower panel of Figure 10). According to our measurements, the angular acceleration of the layer is $g_{\text {angular }}=-(5.4 \pm 1.4) \times 10^{-14} \mathrm{mas} \mathrm{s}^{-2}$. Using Equation (11), this gives a parallax of $5.9 \pm 1.5$ mas. The main source of uncertainty is coming from the estimation of the geometric acceleration of the layer, $g_{\text {angular }}$. 

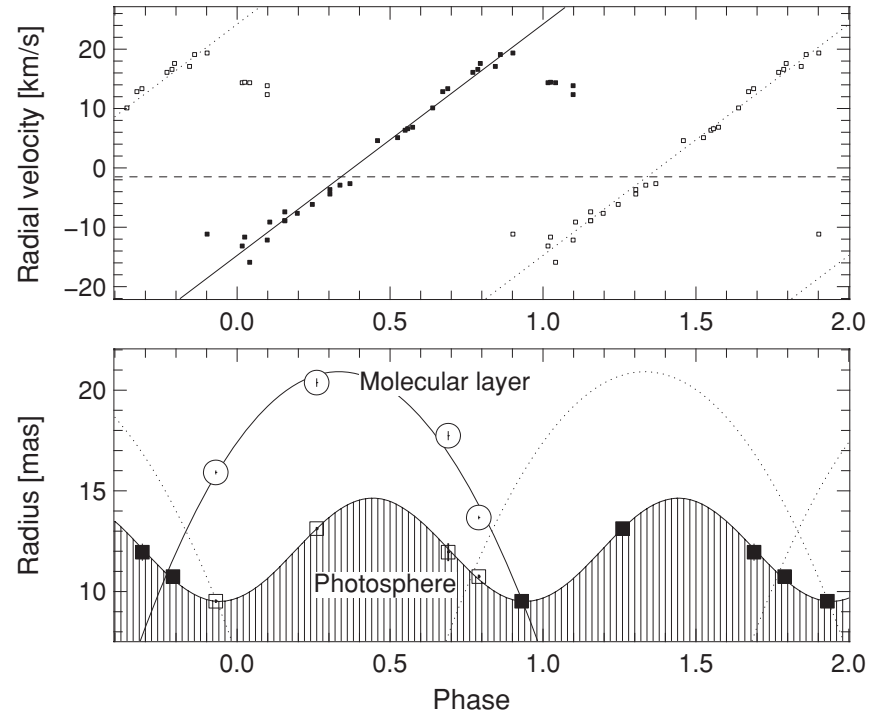

Figure 10. Upper panel: radial velocity of the $\mathrm{CO}(\Delta v=3)$ molecule from Hinkle et al. (1982), folded in phase and shifted by $9.6 \mathrm{~km} \mathrm{~s}^{-1}$ (Wannier et al. 1990). The tilted lines result from a linear fit over the $0<\phi<0.8$ period. It corresponds to a constant acceleration of $g_{\text {radial velocity }}=-(1.10 \pm 0.04) \mathrm{mm} \mathrm{s}^{-2}$. Lower panel: ballistic trajectory of inward acceleration fitted to the position of the molecular layer. The highest point of the ballistic trajectory is at $\phi=0.36$.

Several values of $\chi$ Cyg's parallax exist in the literature, but no consensus exists yet, due to the difficulty of measuring the position of such a star of high angular diameter and inhomogeneous surface brightness. We can cite, among others, a measurement by the Allegheny Observatory $(8.8 \pm 1.9$ mas in Stein 1991), and multiple parallax calculations obtained from the Hipparcos data set: $9.43 \pm 1.36$ mas in Perryman et al. (1997), $6.71 \pm 1.00$ mas in Knapp et al. (2003), and $5.71 \pm 1.12$ mas in Van Leeuwen (2007). Our measurement is compatible with this most recent value. Another way to measure the distance is to use the period/luminosity relation of Mira variables. According to Equation (1), the absolute magnitude of $\chi$ Cyg should be $M_{k}=-8.06 \pm 0.09$. Assuming a $K$ magnitude of -1.95 (including interstellar or circumstellar extinction from Knapp et al. 2003), the distance of $\chi$ Cyg should be 6.1 mas. This last value is also compatible with our measurement.

\subsection{Mass Derivation}

\subsubsection{The Gravitational Field}

Assuming a free-falling molecular layer, the mass of the star can be derived by

$$
M_{\star}=\frac{g R_{\text {layer }}^{2}}{G},
$$

where $g=g_{\text {radial velocity }} / p, G$ is the universal gravitational constant, and $R_{\text {layer }}$ is the position of the molecular layer. Over the period $0<\phi<0.8$, the average radius of the molecular layer is 18 mas, which gives $R_{\text {layer }}=3.0_{-0.6}^{+1.0} \mathrm{AU}$ (for a parallax of $5.9 \pm 1.5 \mathrm{mas})$. Assuming a gravitational acceleration of $g=-(1.37 \pm 0.05) \mathrm{mm} \mathrm{s}^{-2}$, this leads to a stellar mass $M_{\star}=2.1_{-0.7}^{+1.5} M_{\odot}$. Note that the uncertainty on the parallax dominates the error bar.

We are fully aware that the free-fall approximation is an important assumption. Three other forces can affect the trajectory of the molecular layer:

1. pressure force,

2. radiative pressure, and

\section{3. centripetal force due to the rotation of the star.}

The question is whether we can neglect them compared to the gravitational force. If not, our estimate would only yield a lower limit of the mass.

Centripetal force could be dismissed due to the long rotation period of this evolved star. From conservation of momentum energy, it is likely that the star has a period well over several tens of years.

Radiative pressure is responsible for the large mass loss. However, because of the small cross-section between $\mathrm{CO}$ and starlight, the kinetic energy transferred to the molecules would not result in a sensible acceleration. In other words, we expect $\beta_{\mathrm{CO}(\Delta v=3)}$ the ratio of radiation pressure to stellar gravity to be significantly below 1 . A dragging effect could be envisioned, but the velocity gradient between grains and molecules is necessarily small so low in the atmosphere.

Gas pressure force in-between two shocks is likely to be small (Willson 2000). This can be understood as follows: the density gradient of simulated static atmosphere of Mira stars are extremely steep (Bowen 1988). In a static atmosphere, pressure forces induced by density gradient are rigorously equal to the gravitational force. In pulsating Mira stars, the atmosphere is "puffed up," with a density gradient several orders of magnitude smaller. Likewise, pressure forces will be several orders of magnitude lower than the gravitational force (up to a certain distance from the star).

From an observational point of view, this idea is mostly confirmed by the constant acceleration of the $\mathrm{CO}(\Delta v=3)$ molecules. Pressure forces and radiative pressure cannot be constant over the pulsation cycle. Bolometric flux varies by at least a factor 2, and pressure forces may vary even more especially in the case of multiple ballistic trajectories per cycle as proposed by Willson (2006). Thus, if theses forces were dominant, we would detect a nonlinear effect on the acceleration of the molecules. Since the radial velocity measurements of Hinkle et al. (1982) rule out a difference in acceleration of more than $10 \%$ between phase 0 and phase 0.8 , it is unlikely that pressures forces slow the inward acceleration by more than a few percents.

\subsubsection{The Period/Mass/Radius Relation}

Indirect confirmation of $\chi$ Cyg mass is difficult. Indeed, $2.1 M_{\odot}$ is quite massive for a Mira star (Vassiliadis \& Wood 1993). However, $\chi$ Cyg may not be a Mira of the most common sort. First, the star has often been noticed by the remarkable strength of his emission lines (e.g., Merrill 1947). Second, its 408 day period is slightly longer than the average pulsation period of Mira stars (Whitelock et al. 2000). Lastly, according to our parallax measurement, its mean radius of $440_{-50}^{+150} R_{\odot}$ (12.1 mas) makes $\chi$ Cygni a quite huge object.

The feeling that $\chi$ Cygni can be a quite massive Mira star is confirmed by the $\mathrm{P} / \mathrm{M} / \mathrm{R}$ relation. Indeed, applying $\chi$ Cyg's parameters to Equation (2) gives a mass of $3.1_{-1.2}^{+2.7} M_{\odot}$. This value is compatible with our measurement.

\subsection{Discussion}

This work makes use of several assumptions which will certainly need to be fine tuned. If anything, they emphasize the need to complete the method with detailed numerical modeling to assess the influence of atmospheric pressure forces. In the case of $\chi$ Cyg, the consistency of the acceleration was the main argument to neglect pressure effects. We also neglected 
the influence of an eventual centrifugal force as it is unlikely because of the slow rotation of the star's atmosphere. Finally, we assumed the molecular layer detected in the interferometric data to be at the same position as the $\mathrm{CO}(\Delta v=3)$ molecules observed by Hinkle et al. (1982). This last point may be the most arguable one. Even though both spectroscopic and interferometric observations have been made in the $H$ band, it is true that contamination may exist due to molecules (e.g., $\mathrm{H}_{2} \mathrm{O}$ ) possibly formed at a slightly different height inside the atmosphere. Clear distinction between several possible molecular layers will nevertheless be possible in the near future thanks to high resolution spectro-interferometry. By probing the apparent stellar diameter within the depth of saturated absorption lines, it would provide the velocity and the position for individual species inside the atmosphere. This is a prospect which would not only strengthen our mass and distance determination method, but also would be bound to revolutionize our understanding of shock-driven atmospheres.

Evolved stars could prove to be precious secondary distance indicators. Compared to Cepheid, the fact that these stars are large and extremely bright in the infrared (where interstellar absorption is minimal), would make them a precious secondary indicator. To allow that, the $\mathrm{P} / \mathrm{L}$ relation of LPV stars should be properly assessed. This work is a first step in that direction. Moreover, this method has an intrinsic advantage compared to the traditional Baade-Wesselink method since the projection factor can be derived from the position of the molecular layer (even though we agree that the projection factor is not yet the limiting term in the final accuracy of this method).

Deriving the mass of these stars also opens an important prospect to study stellar structure, chemistry, and mass loss. It is one of the main parameters (with radius) to constrain stellar instabilities and oscillation modes. This novel way to derive the mass is applicable to a wide variety of pulsating objects. The requirement is to be able to probe a portion of the atmosphere where the gravitational field is significantly stronger than the pressure forces (conventional and radiative). It only happens in shock-driven atmospheres, but nevertheless should be observable in numerous types of pulsating stars. For example, recent results on Cepheids showed a faint upper atmospheric molecular layer likely to be gravitationally unstable (Mérand et al. 2006b). Note that this technique does not require a regular pulsating variable; it can also be applied to any irregular or semiregular variables, featuring shock-driven molecular layers.

We acknowledge with thanks the variable star observations from the AAVSO International Database contributed by observers worldwide and used in this research. S.T.R. acknowledges partial support from NASA grant NNH09AK731.

\section{REFERENCES}

Andrievsky, S. M., Luck, R. E., \& Kovtyukh, V. V. 2005, AJ, 130, 1880

Baade, W. 1926, Astron. Nachr., 228, 359

Barthes, D. 1998, A\&A, 333, 647

Berger, J.-P., et al. 2003, Proc. SPIE, 4838, 1099

Bertschinger, E., \& Chevalier, R. A. 1985, ApJ, 299, 167

Bessell, M. S., Scholz, M., \& Wood, P. R. 1996, A\&A, 307, 481

Bordé, P., Coudé du Foresto, V., Chagnon, G., \& Perrin, G. 2002, A\&A, 393, 183

Bowen, G. H. 1988, ApJ, 329, 299

Claret, A. 2000, A\&A, 363, 1081

Davis, J., Tango, W. J., \& Booth, A. J. 2000, MNRAS, 318, 387

Hestroffer, D. 1997, A\&A, 327, 199

Hinkle, K. H. 1978, ApJ, 220, 210
Hinkle, K. H. 1995, in ASP Conf. Ser. 83, IAU Colloq. 155: Astrophysical Applications of Stellar Pulsation, ed. R. S. Stobie \& P. A. Whitelock (San Francisco, CA: ASP), 399

Hinkle, K. H., Hall, D. N. B., \& Ridgway, S. T. 1982, ApJ, 252, 697

Hinkle, K. H., Lebzelter, T., \& Scharlach, W. W. G. 1997, AJ, 114, 2686

Hinkle, K. H., Scharlach, W. W. G., \& Hall, D. N. B. 1984, ApJS, 56, 1

Hofmann, K.-H., Scholz, M., \& Wood, P. R. 1998, A\&A, 339, 846

Ireland, M. J., Scholz, M., Tuthill, P. G., \& Wood, P. R. 2004a, MNRAS, 355, 444

Ireland, M. J., Scholz, M., \& Wood, P. R. 2004b, MNRAS, 352, 318

Ireland, M. J., Scholz, M., \& Wood, P. R. 2008, MNRAS, 391, 1994

Jacob, A. P., \& Scholz, M. 2002, MNRAS, 336, 1377

Kervella, P., Nardetto, N., Bersier, D., Mourard, D., \& Coudé du Foresto, V. 2004, A\&A, 416, 941

Knapp, G. R., Pourbaix, D., Platais, I., \& Jorissen, A. 2003, A\&A, 403, 993

Lacour, S., et al. 2008, A\&A, 485, 561

Le Besnerais, G., Lacour, S., Mugnier, L. M., Thiebaut, E., Perrin, G., \& Meimon, S. 2008, IEEE J. Sel. Top. Signal Process., 2, 767

Le Bouquin, J.-B., Lacour, S., Renard, S., Thiébaut, E., Merand, A., \& Verhoelst, T. 2009, A\&A, 496, L1

Lebzelter, T., Hinkle, K. H., Wood, P. R., Joyce, R. R., \& Fekel, F. C. 2005, A\&A, 431,623

Lebzelter, T., \& Wood, P. R. 2005, A\&A, 441, 1117

Matsunaga, N., Kawadu, T., Nishiyama, S., Nagayama, T., Hatano, H., Tamura, M., Glass, I. S., \& Nagata, T. 2009, MNRAS, 399, 1709

Mennesson, B., et al. 2002, ApJ, 579, 446

Mérand, A., Bordé, P., \& Coudé Du Foresto, V. 2006a, A\&A, 447, 783

Mérand, A., et al. 2006b, A\&A, 453, 155

Merrill, P. W. 1947, ApJ, 106, 274

Michelson, A. A., \& Pease, F. G. 1921, ApJ, 53, 249

Monnier, J. D., et al. 2006, ApJ, 647, 444

Ohnaka, K. 2004, A\&A, 424, 1011

Paladini, C., Aringer, B., Hron, J., Nowotny, W., Sacuto, S., \& Hoefner, S. 2009, A\&A, 501, 1073

Pauls, T. A., Young, J. S., Cotton, W. D., \& Monnier, J. D. 2005, PASP, 117, 1255

Pedretti, E., et al. 2008, Proc. SPIE, 7013, 88

Perrin, G., Coude Du Foresto, V., Ridgway, S. T., Mariotti, J.-M., Traub, W. A., Carleton, N. P., \& Lacasse, M. G. 1998, A\&A, 331, 619

Perrin, G., Coudé du Foresto, V., Ridgway, S. T., Mennesson, B., Ruilier, C., Mariotti, J.-M., Traub, W. A., \& Lacasse, M. G. 1999, A\&A, 345, 221

Perrin, G., et al. 2004, A\&A, 426, 279

Perryman, M. A. C., et al. 1997, A\&A, 323, L49

Pluzhnik, E. A., Ragland, S., LeCoroller, H., Cotton, W. D., Danchi, W. C., Traub, W. A., \& Willson, L. A. 2009, ApJ, 700, 114

Reid, M. J., \& Menten, K. M. 1997, ApJ, 476, 327

Rejkuba, M. 2004, A\&A, 413, 903

Scholz, M. 2003, Proc. SPIE, 4838, 163

Scholz, M., \& Takeda, Y. 1987, A\&A, 186, 200

Stein, J. W. 1991, ApJ, 377, 669

Strecker, D. 1973, PhD thesis, University of Minnesota

Thiébaut, E. 2008, Proc. SPIE, 7013, 43

Thiébaut, E., Garcia, P. J. V., \& Foy, R. 2003, Ap\&SS, 286, 171

Thiébaut, É., \& Giovannelli, J.-F. 2009, IEEE Signal Process. Mag., in press

Thompson, R. R., Creech-Eakman, M. J., \& Akeson, R. L. 2002, ApJ, 570, 373

Traub, W. A., et al. 2003, Proc. SPIE, 4838, 45

Tsuji, T. 2000, ApJ, 540, L99

van Belle, G. T., Dyck, H. M., Benson, J. A., \& Lacasse, M. G. 1996, AJ, 112 2147

Van Leeuwen, F. 2007, Hipparcos, the New Reduction of the Raw Data (Berlin: Springer)

Vassiliadis, E., \& Wood, P. R. 1993, ApJ, 413, 641

Wannier, P. G., Sahai, R., Andersson, B.-G., \& Johnson, H. R. 1990, ApJ, 358 251

Weiner, J., Hale, D. D. S., \& Townes, C. H. 2003, ApJ, 589, 976

Wesselink, A. J. 1946, Bull. Astron. Inst. Netherlands, 10, 91

Whitelock, P. A., Feast, M. W., \& van Leeuwen, F. 2008, MNRAS, 386, 313

Whitelock, P., Marang, F., \& Feast, M. 2000, MNRAS, 319, 728

Willson, L. A. 2000, ARA\&A, 38, 573

Willson, L. A. 2006, J. Am. Assoc. Variable Star Observers, 35, 62

Wittkowski, M., Aufdenberg, J. P., Driebe, T., Roccatagliata, V., Szeifert, T., \& Wolff, B. 2006, A\&A, 460, 855 
Wittkowski, M., Boboltz, D. A., Driebe, T., Le Bouquin, J.-B., Millour, F., Ohnaka, K., \& Scholz, M. 2008, A\&A, 479, L21

Wood, P. R. 1990, in From Miras to Planetary Nebulae: Which Path for Stellar Evolution, ed. M. O. Mennessier \& A. Omont (Gif-sur-Yvette: Editions Frontieres), 67

Wood, P. R. 2000, PASA, 17, 18
Woodruff, H. C., Tuthill, P. G., Monnier, J. D., Ireland, M. J., Bedding, T. R., Lacour, S., Danchi, W. C., \& Scholz, M. 2008, ApJ, 673, 418

Ya'Ari, A., \& Tuchman, Y. 1999, ApJ, 514, L35

Young, J. S., et al. 2000, MNRAS, 318, 381

Zijlstra, A. A. 1999, in IAU Symp. 191, Asymptotic Giant Branch Stars, ed. T. Le Bertre, A. Lebre, \& C. Waelkens (Dordrecht: Kluwer), 551 